\title{
Should we do battle with antivaccination activists?
}

\author{
Julie Leaska,b \\ a School of Public Health, University of Sydney, NSW, Australia \\ b Corresponding author: Julie.Leask@sydney.edu.au
}

\section{Article history}

Publication date: March 2015

Citation: Leask J. Should we do battle with antivaccination activists? Public Health Res Pract. 2015;25(2):e2521515. doi: http:// dx.doi.org/10.17061/phrp2521515

\section{Key points}

- Adversarial approaches against opponents of public health have resulted in several achievements, including better regulation of harmful products

- Antivaccination activists have sought to dissuade people from vaccinating themselves or their children

- Some advocates of vaccination adopt or encourage adversarial approaches against such activists. However, these approaches can give antivaccination activists more oxygen, reinvigorating highly polarised debates that may alienate vaccine-hesitant parents

- Adversarial approaches do not sufficiently account for, nor address, parents' complex decision mechanisms

- In vaccination advocacy, it is generally better to play the issue, not the opponent

\section{Abstract}

Antivaccination activists have existed since variolation was introduced in Europe in the 18th century. Today, they continue to attempt to influence the vaccination decisions of parents. Commentators have expressed concern about the impact of such activists on vaccination rates and disease outbreaks. Some argue that public health advocates should engage in adversarial approaches involving public attempts to discredit or stop an antivaccination group or individual.

This article argues that such adversarial advocacy may not be the most effective way to support vaccine programs. It argues this on the basis of what is known to influence vaccination attitudes and uptake, and the unintended negative consequences that can arise from an adversarial approach. These include drawing attention to such activists and their arguments, and potentially alienating the most important audience - hesitant parents - where the primary goal is to establish trust. The exception is when the antivaccination activists' actions may cause direct harm, such as encouraging a 'disease party' or illegal activities.

Generally, however, advocacy should focus on areas where real gains can be made - on policies that directly address determinants of low coverage such as lack of opportunity to vaccinate and lack of acceptance of vaccination. This includes advocacy for accessible and affordable vaccines. In addressing the global problem of vaccine hesitancy, public health has a responsibility to better monitor public attitudes, support health professionals in communication, and develop and test strategies that engage vaccine-hesitant parents and communities.

\section{Introduction}

In 1877, renowned children's author Lewis Carroll found himself embroiled in a newspaper debate about vaccination. ${ }^{1}$ Carroll had read a letter published in the Eastbourne Chronicle in which Mr W Hume-Rothery claimed that the smallpox vaccine was causing smallpox in large numbers of people. Using his real name of Charles $L$ Dodgson, Carroll refuted the claims with his characteristic eloquence. This correspondence escalated into an increasingly 
defensive exchange between Carroll and Hume-Rothery, in which Carroll, "a trifle ruffled but keeping to the point, retired after the third round". His opponent continued vigorously until the editor ended the correspondence.

Opposition to vaccination, as expressed within such public debates, has occurred since variolation was introduced in Europe in the 18th century. Today, organised opposition to vaccination is a worldwide phenomenon. The manifest claims of opponents centre on the belief that vaccines are unsafe and ineffective. Activists promote themselves as champions of transparency in public information and individual choice, and locate their rhetoric within latent themes of cover-up, manipulation for profit, threat of excessive government control and the back-to-nature idyll. ${ }^{2}$

Hobson-West characterised two types of 'vaccinecritical' groups: 'reformists', who were critical of vaccines but likely to support vaccination in some respects, and 'radicals', who questioned the rationale for, and use of, all vaccines. ${ }^{3}$ Australia's main 'vaccine critical group', the Australian Vaccination Skeptics Network, is a radical group whose 1998 book asserts that "vaccines are extremely toxic and are suspected to cause more harm to more vaccinees than results when infection is naturally acquired". ${ }^{4}$ It claims, for example, that smallpox, which was declared eradicated by the World Health Organization in 1980, "has enormous non-human reservoirs in which it continues to survive and infect humans, but those cases of smallpox which continue to occur are 'creatively diagnosed' and reported under another name". The book lists homeopathy, reflexology, chiropractic, traditional Chinese medicine and a good diet as possible alternatives to vaccination.

Such groups are mostly made up of parents. ${ }^{5}$ Some may express an underlying antipathy to medical intervention, with a preference for natural or alternative modes of disease prevention and management. Among these parents, nonvaccination is bound up with how they shape their individual identity as vigilant parents questioning the medical status quo, and their group commitments. Not vaccinating is a manifestation of a deeper set of shared values. Some parents have at one time supported vaccination, but one of their children has experienced a frightening but temporary adverse event or a chronic, permanent and usually idiopathic condition, which they believe to be a result of vaccination. They then embark on phases of questioning and decision making. ${ }^{6}$ Other activists include general citizens, some health professionals, and providers of alternative therapies. ${ }^{7}$

Some commentators lament the impact of antivaccination activist groups. In a recent article, Garrett and Builder argued that "misinformation and rumours from just one persuasive voice, delivered effectively, can derail entire immunisation campaigns and persuade millions of parents to shun vaccinations for their children". ${ }^{8}$ Citing the contribution of antivaccination groups to outbreaks of disease, a 2011 editorial in the New England Journal of
Medicine asked "what can we do to hasten the funeral of anti-vaccination campaigns?"9

Some of public health's greatest achievements have been won through adversarial advocacy, either by reducing the credibility of an opponent's messages or by contributing to regulations that limit the reach and adverse consequences of their products. Adversarial public advocacy has contributed to achievements in tobacco control, harmful drinking reduction and gun control.

Should public health and other professionals use adversarial advocacy against antivaccination activists to minimise their potential impact? Here, adversarial advocacy is defined as attempts to publicly discredit or disband an antivaccination activist or group. Such an approach might seem reasonable under two assumptions. The first is that the claims of the antivaccination activists directly and significantly contribute to undervaccination. Second is the assumption that such advocacy could succeed in stopping the movement in general. Both assumptions have flaws.

\section{The role of antivaccination activist groups in the vaccine decisions of parents}

It is often assumed that audiences of naïve parents uncritically absorb antivaccination messages - the more messages, the more vaccine fear and the less vaccination. In the early 1970s, media scholars largely discredited this model of media influence, pointing to the social and cultural contexts in which audiences decoded messages and derived meaning. ${ }^{10} \mathrm{~A}$ case in point is the UK's measles-mumps-rubella (MMR) vaccine scare, which saw the theory of a link to autism catch on despite evidence to the contrary. Many parents lost confidence in the vaccine, resulting in an 11\% drop in UK MMR vaccination rates and a measles epidemic. The impact was not immediate. Groups had previously linked vaccines to autism, but this scare had a charismatic doctor as its champion, whose (eventually retracted) publication in The Lancet meant that mainstream journalists took note. Aside from a medical champion, a number of other factors unique to the UK were at play. There was some erosion of health professional confidence in the vaccine. The government's previous handling of the Creutzfeldt-Jakob disease affair provided a foundation of mistrust in government safety assurances. A sustained anti-MMR campaign gained momentum in the tabloid press, which was amplified by prime minister Tony Blair's refusal to acknowledge whether or not his son Leo had been given MMR. ${ }^{11}$

Journalists could readily access moving narratives from parents who were convinced that their child's autism resulted from the vaccine. Sometimes, members of antivaccination groups facilitated this access and were 
also given airtime. However, their role in eroding MMR vaccination rates was one of many amplifiers.

Australia was relatively insulated from the effects of the MMR scare, despite attempts by local activists to amplify it through media releases, background media lobbying, websites and social media. ${ }^{7}$ Many parents presented their concerns about MMR to health professionals, but national vaccination rates remained stable at around $92 \% .{ }^{12}$ This stability has been maintained through two decades of activity from a small but active and vociferous antivaccination group and a concomitant rise in audience exposure to vaccine-critical messages through the internet and social media. It reinforces that antivaccination groups and activists act within a complex set of influencers.

\section{Antivaccination groups persist and attempts to silence them may amplify exposure to their messages}

It is unrealistic to believe that it is possible to cease antivaccination efforts. As long as vaccination has existed, there have been such activists, just as there will always be a minority who stand outside the mainstream, reject orthodox medicine and its interventions, mistrust government and value natural health. Radical activist groups tend to be self-limiting in their appeal because of that radicalism. ${ }^{13}$ With the reformist groups, robust vaccine programs in democratic societies can and should be able to tolerate the existence of critics. Such programs come with coordinated adverse events reporting systems that are underpinned by the willingness to detect, investigate and respond to safety signals. ${ }^{14}$

Radical antivaccination groups, unlike industry bodies, are difficult to regulate. They arise organically, consisting of small core groups of highly motivated and vocal individuals who devote large amounts of time and energy to their cause. As for other such movements, a highly adversarial strategy could give oxygen to antivaccination activists, who may believe that persecution legitimises their efforts within a martyrdom frame. It also affords more attention to them, stimulating highly polarised discussions in social and traditional media, and perpetuating a false sense that vaccination is a highly contested topic.

Typically, adversarial approaches have been effective where they are directed towards industries or groups who promote a harmful product. With vaccination, public health advocates promote the product and opponents are often parents with moving stories of disabled or ill children. In the media, the primary contest is one of establishing the trust of hesitant-parent audiences and reminding them of why we vaccinate. Adversarial approaches can engender mistrust if they show advocates as overly defensive and lacking empathy.

\section{Playing the issue, not the opponent}

What, then, is the best focus of vaccine advocacy for time- and resource-limited public health professionals? It should be on addressing the causes of low coverage where two demographically distinct groups are seen: 1) those who lack opportunity to vaccinate and face practical, economic, social or geographic impediments to full and timely vaccination; and 2) those who lack acceptance of vaccination and have beliefs, attitudes and experiences that cause them to reject or delay some or all vaccines. They may worry that vaccines have harmful ingredients, can weaken the immune system of the baby and that children get too many vaccines. ${ }^{15}$

For parents lacking opportunity, systematic reviews underscore the need to advocate for recommended vaccines to not incur out-of-pocket expenses and be easily accessible; for accurate national recording; for incentives; and for reminders for parents and providers. ${ }^{16,17}$ Specific strategies are needed for Indigenous and culturally and linguistically diverse families to ensure services are culturally respectful, easy to access and flexible.

Among those who lack acceptance, parents who are highly hesitant about vaccination (not entrenched nonvaccinators) warrant the greatest investment. This includes supporting health professionals in their communication, ensuring that tailored and effective information is readily available, and establishing routine monitoring of vaccination attitudes to provide early warning of a dip in order to guide timely action.

Countries that have recovered from vaccine safety scares, such as Nigeria, teach advocates that community understanding and health diplomacy with influential leaders is effective. ${ }^{18}$ When vaccine safety scares arise, whether they are real or perceived, it is ideal for governments to have some preparation in place. This includes plans for who will communicate and when, and established channels of communication to providers in particular, helping them to be confident and competent to address the concerns of parents.

\section{Should advocates always ignore the antivaccination activists?}

There are occasions warranting direct action with antivaccination activists or groups. These include when advice or activities could lead to direct and immediate harm to individuals, such as the promotion of infectious disease parties, harassment or threats. In addition, it may be warranted to stop their intrusion into public institutions (such as distributing leaflets in schools) where the setting could markedly increase the perceived legitimacy of their messages. 


\section{Conclusion}

Vaccination has prevented illness and death in millions of children and adults. ${ }^{19}$ It is understandable that advocates sometimes passionately want to defend vaccination programs. However, advocates need to be strategic, working in with the known mechanisms through which uptake can be improved. This includes advocacy for accessible and affordable vaccines. With respect to media audiences, vaccine-hesitant parents are the most important group. For them, trust in the source of information is a powerful moderator of message influence. Publicly adversarial debates are unlikely to convince them to vaccinate and may merely serve as catharsis for the converted. Advocates can employ empathic responses that focus on the issue, not the opponent, and appropriate the values underscoring vaccination - protection of children from serious diseases with an explicit acknowledgement of the importance of vaccine safety.

\section{Acknowledgements}

The author would like to thank Ken Griffin for comments on an earlier draft, and two anonymous peer reviewers.

\section{Competing interests}

The author has been a consultant to the European Office of the World Health Organization. She has received lecture fees from Medicare Locals; travel expenses from the Public Health Association of Australia related to this article; and travel expenses from Fondation Mérieux not related to this article.

\section{References}

1. Crutch D, editor. Lewis Carroll: three letters on antivaccination (1877). London: Lewis Carroll Society; 1976.

2. Leask JA, Chapman S. An attempt to swindle nature: press anti-immunisation reportage 1993-1997. Aust NZ J Public Health. 1998;22(1):17-26.

3. Hobson-West P. Trusting blindly can be the biggest risk of all: organised resistance to childhood vaccination in the UK. Sociol Health IIIn. 2007;29(2):198-215.

4. Australian Vaccination Network. Vaccination roulette: experiences, risks and alternatives. Bangalow (NSW): Australian Vaccination Network; 1998.
5. Wilson T. A profile of the Australian Vaccination Network 2012 [Internet]. Bangalow (NSW): Australian Vaccination Network; 2013 [cited 2014 Nov 6]. Available from: www.nocompulsoryvaccination.com/2013/07/04/avnmembership-well-educated-and-well-informed/

6. Davies EA, Blair A. Parental reasons for discontinuing the Australian vaccination schedule. Neonatal, Paediatric \& Child Health Nursing. 2003;6(1):6-12.

7. Leask J. Understanding immunisation controversies [dissertation]. [Sydney]: University of Sydney; 2002. Available from: ses.library.usyd.edu.au/ handle/2123/12503

8. Garrett L, Builder M. Vaccine conspiracy theories are deadly, contagious. LA Times. Oct 232014.

9. Poland GA, Jacobson RM. The age-old struggle against the antivaccinationists. N Engl J Med. 2011;364(2):97-9.

10. Hall S. Encoding and decoding in the television discourse. Birmingham: University of Birmingham; 1973.

11. Goldacre B. Bad science. London: Fourth Estate; 2008.

12. Leask J, Booy R, McIntyre PB. MMR, Wakefield and The Lancet: what can we learn? Med J Aust. 2010;193(1):5-7. Erratum in: Med J Aust. 2010;193(3):192.

13. Leask J, Chapman S, Hawe P, Burgess M. What maintains parental support for vaccination when challenged by anti-vaccination messages? A qualitative study. Vaccine. 2006;24(49-50):7238-45.

14. Cooper LZ, Larson HJ, Katz SL. Protecting public trust in immunization. Pediatrics. 2008;122(1):149-53.

15. Brown KF, Kroll JS, Hudson MJ, Ramsay M, Green J, Long SJ, et al. Factors underlying parental decisions about combination childhood vaccinations including MMR: a systematic review. Vaccine. 2010;28(26):4235-48.

16. Ward K, Chow MYK, King C, Leask J. Strategies to improve vaccination uptake in Australia, a systematic review of types and effectiveness. Aust N Z J Public Health. 2012;36(4):369-77.

17. Community Preventive Services Task Force (US). The community guide: increasing appropriate vaccination. Washington: US Department of Health and Human Services; 2014 Jun [cited 2012 Sep 1]. Available from: www.thecommunityguide.org/vaccines/index.html

18. Kaufmann JR, Feldbaum H. Diplomacy and the polio immunization boycott in Northern Nigeria. Health Aff (Millwood). 2009;28(4):1091-101.

19. Ozawa S, Stack ML, Bishai DM, Mirelman A, Friberg IK, Niessen $L$, et al. During the 'decade of vaccines', the lives of 6.4 million children valued at $\$ 231$ billion could be saved. Health Aff (Millwood). 2011;30(6):1010-20.

\section{Copyright: (c) () ()}

(c) 2015 Leask. This article is licensed under the Creative Commons Attribution-NonCommercial-ShareAlike 4.0 International Licence, which allows others to redistribute, adapt and share this work non-commercially provided they attribute the work and any adapted version of it is distributed under the same Creative Commons licence terms. See: www.creativecommons.org/licenses/by-nc-sa/4.0/ 